Pacific Journal of Mathematics

PRINCIPLES OF PARTIAL REFLECTION IN THE SET
THEORIES OF ZERMELO AND ACKERMANN 


\title{
PRINCIPLES OF PARTIAL REFLECTION IN THE SET THEORIES OF ZERMELO AND ACKERMANN
}

\author{
A. LÉvy AND R. VAUGHT
}

It was shown in [4] that the Zermelo-Fraenkel set theory may be obtained by adjoining to the Zermelo theory $\boldsymbol{Z}$ an axiom schema called the principle of complete reflection. (This schema, denoted here by $\boldsymbol{C R}$, and other notions involved in these introductory remarks will be described explicitly later.) A schema of partial reflection, called here $\boldsymbol{P R}$, which is also valid in $\boldsymbol{Z F}$ was described in [5]. As we shall see in $\S 2$, the theory $\boldsymbol{T}_{0}=\boldsymbol{Z}+\boldsymbol{P R}$ is a very strong one, in which, apparently, all of the 'ordinary' set-theoretical constructions can be carried out.

Nevertheless, we shall show in $\S 4$ that $\boldsymbol{T}_{0}$ is much weaker than $\boldsymbol{Z F}$. Indeed, we shall prove within $\boldsymbol{Z F}$ the existence of numerous wellbehaved models of a theory $\boldsymbol{T}_{1}$ which deals with classes as well as sets and is based on a partial reflection principle $\boldsymbol{P} \boldsymbol{R}_{1}$ stronger than $\boldsymbol{P R}$.

The theory $\boldsymbol{A}^{*}$ of [3], which is the set theory of Ackermann [1] with an axiom of regularity added, deals with classes as well as set (members of $V$ ). Regarding its 'purely set-theoretical' part $\boldsymbol{A}^{*} / V$, it is known that $\boldsymbol{A}^{*} / V \subseteq \boldsymbol{Z F}$ (cf. [3]). An interesting question, which remains open, is whether $\boldsymbol{A}^{*} / V$ and $\boldsymbol{Z} \boldsymbol{F}$ coincide. The development of the theory $\boldsymbol{A}^{*}$ has already been carried out in [1] and [3] to a considerable extent. In particular, it was shown in [3] that $\boldsymbol{T}_{0} \subseteq \boldsymbol{A}^{*} / V$ and, moreover, that another sort of partial reflection principle, called here $\boldsymbol{R}$, is valid in $\boldsymbol{A}^{*}$. As we shall observe below, the central axiom schema $(\gamma)$ of $\boldsymbol{A}^{*}$ may, in fact, be replaced by $\boldsymbol{R}$.

In order to investigate the strength of $\boldsymbol{A}^{*}$, we shall, in $\S 6$, establish within $\boldsymbol{A}^{*}$ the same facts concerning the existence of models of $\boldsymbol{T}_{1}$, which have been previously proved in $\S 4$ on the basis of $\boldsymbol{Z F}$. The very short proofs within $\boldsymbol{Z F}$ of these facts depend heavily on the axiom of replacement, or that of complete reflection. In order to establish the same facts within $\boldsymbol{A}^{*}$ we are forced to carry further the general development of $\boldsymbol{A}^{*}$. In particular various results will be obtained within $\boldsymbol{A}^{*}$ concerning proper classes, which have hitherto been little investigated.

§1. Preliminaries. Each set theory $\boldsymbol{T}$ we shall consider is formalized in the first order logic with identity and has as its only nonlogical symbols the binary predicate $\varepsilon$ plus, in some cases, the individual constant $V$. Its only sentential connective is $\mid$ (joint denial) and its only quantifier is $\exists$. (The symbols ' $\wedge$ ', ' $\forall$ ', etc., used in the metalanguage

Received August 2, 1960. This work was supported by the National Science Foundation under Grant G-8934. 
are to be understood in the usual way.) The words 'theory', 'formula', 'valid' etc., are to be understood as in [8]. We shall, however, say that a formula is valid in $\boldsymbol{T}$ provided that its universal closure is a sentence valid in $\boldsymbol{T}$. Moreover, each theory has a specified set of axioms. If $\boldsymbol{T}$ is a set theory and $\boldsymbol{X}$ is a sentence or a sentence schema, we denote by $\boldsymbol{T}+\boldsymbol{X}$ the theory whose axioms are all axioms of $\boldsymbol{T}$ together with the sentence or sentences $X$. We write $\boldsymbol{T} \subseteq T^{\prime}$ to mean that every sentence valid in the theory $\boldsymbol{T}$ is valid in $\boldsymbol{T}^{\prime}$.

Throughout the paper we shall use the letters $\theta, \phi$, and $\psi$, or $\sigma$ to range over formulas or sentences, respectively, involving only $\varepsilon$, and $\Theta$, $\Phi$, and $\Psi$, or $\Sigma$ or $\tau$ to range over formulas or sentences or terms respectively, which may involve $V$ as well. Concatenation will be denoted by juxtaposition. We write $\vdash_{T} \Phi(\vdash \Phi)$ to mean that $\Phi$ is valid in the theory $\boldsymbol{T}$ (logically valid).

It is assumed that the distinct variables (of any theory) are $v_{0}, v_{1}, \cdots$. To improve readability we agree that $x, y, z, u$ are in order $v_{0}, v_{1}, v_{2}, v_{3}$ and that $x, y, z, u, v, w, s, t, x^{\prime}, x_{0}, \cdots, y^{\prime}, \cdots$ are distinct variables. If $\tau_{0}, \cdots, \tau_{n-1}$ are terms we denote by $\Phi\left(\tau_{0}, \cdots, \tau_{n-1}\right)$ the formula obtained from $\Phi$ by simultaneously substituting $\tau_{i}$ for $v_{i}(i<n)$ - after first changing bound variables in $\Phi$ to avoid any collisions.

We denote by $\left(\exists v_{k_{0}}, v_{k_{1}}, \cdots, v_{k_{n}} \in \tau\right) \Phi$ the formula

$$
\left(\exists v_{k_{0}}\right) \cdots\left(\exists v_{k_{n}}\right)\left(v_{k_{0}} \in \tau \wedge \cdots \wedge v_{k_{n}} \in \tau \wedge \Phi\right) .
$$

Two or three other similar usages will be observed which are to be understood analogously.

By $\operatorname{Rel}(\tau, \Phi)$ we mean the formula obtained from $\Phi$ by relativizing its quantifiers to $\tau$, i.e., by replacing each part of the form $\left(\exists v_{k}\right) \Theta$ by $\left(\exists v_{k} \varepsilon \tau\right) \Theta$, after first changing bound variables to avoid collisions.

Let $\boldsymbol{T}$ be a set theory involving $V$. In the course of proofs within $\boldsymbol{T}$ which are being approximated in English, we shall refer to arbitrary objects as classes and to members of $V$ as sets. We denote by $\boldsymbol{T} / V$ the theory without the constant $V$ whose axioms (and whose valid sentences) are all sentences $\sigma$ such that $\vdash_{T} \operatorname{Rel}(V, \sigma)$.

The following special formulas will be needed.

DEFINITION 1.
$x \subseteq y$ for
$(\forall z)(z \in x \rightarrow z \in y)$
$C p(x)$ for
$(\forall y, z)(z \in y \wedge y \in x \rightarrow z \in x)$,
read ' $x$ is complete'
$S c(x)$ for $C p(x) \wedge$
$(\forall y, z)(z \subseteq y \wedge y \in x \rightarrow z \in x)^{(1)}$

read ' $x$ is supercomplete'

1 Strictly, we should say that $S c$ (rather than $S c(x)$ ) is the formula on the right. In addition, we are agreeing to write $v_{k} \subseteq v_{l}$ instead of $\subseteq\left(v_{k}, v_{l}\right)$. 
$\operatorname{Ord}(x)$ for $C p(x) \wedge(\forall y, z, w \in x)[y \notin y \wedge(y \in z \vee y=z \vee z \in y)$

$\wedge(w \in y \wedge y \in z \rightarrow w \in z)] \wedge(\forall u)[u \subseteq x \wedge(\exists t)(t \in u) \rightarrow(\exists t) t \in u$

$\wedge \sim(\exists s)(s \in t \wedge s \in u))] \wedge(\forall u, v \subseteq x)(\exists w)(\forall t)[t \in w \longleftrightarrow t \in u$

$\wedge t \notin v]$

read ' $x$ is an ordinal'

$D b(x, y, z)$ for $(\forall t)(t \in z \longleftrightarrow t=x \vee t=y)$ read ' $z$ is the doubleton $\{x, y\}$ '

$O c(x, y, z)$ for $(\exists v, w)(D b(x, x, v) \wedge D b(x, y, w) \wedge D b(v, w, z))$

read ' $z$ is the ordered couple $\langle x y>$ '

$R l(x, y, z)$ for $(\exists v)(O c(x, y, v) \wedge v \in z) \quad \operatorname{read}$ ' $x$ is in the relation $z$ to $y$ ' $R n(x, y)$ for $\operatorname{Ord}(x) \wedge(\exists z)\{(\forall u)(u \in x \rightarrow(\exists v) R l(u, v, z))$

$\wedge(\forall u, v)[(u \in x \wedge R l(u, v, z)) \vee(u=x \wedge v=y)$

$\left.\left.\rightarrow(\forall t)\left(t \in v \longleftrightarrow\left(\exists u^{\prime}, v^{\prime}\right)\left(u^{\prime} \in u \wedge R l\left(u^{\prime}, v^{\prime}, z\right) \wedge t \subseteq v^{\prime}\right)\right)\right]\right\}$

read ' $x$ is an ordinal and $R(x)$ exists and equals $y^{\prime(2)}$

$W(x)$ for $(\exists u)[R n(u, x) \wedge(\forall v \in u)(\exists \mathrm{w} \in \mathrm{u})(v \in w) \wedge(\exists y)(y \in u)]$

read 'For some limit ordinal $\lambda, R(\lambda)$ exists and equals $x$ '.

The theories $\boldsymbol{S}_{0}, \boldsymbol{S}, \boldsymbol{Z}$ and $\boldsymbol{Z F}$ have no individual constants. $\boldsymbol{S}_{0}$ is based upon the axiom of extensionality $x \subseteq y \wedge y \subseteq x \rightarrow x=y$, the axiom schema of subsets

$$
(\exists x)(\forall y)(y \in x \longleftrightarrow y \in z \wedge \varphi) \quad(x \text { not free in } \varphi)
$$

and the axiom of regularity

$$
(\exists y)(y \in x) \rightarrow(\exists y)[y \in x \wedge \sim(\exists z)(z \in y \wedge z \in x)] .
$$

$\boldsymbol{S}$ has, in addition, the axioms of pairing, sum set and power set, and the axiom schema of regularity. $\boldsymbol{Z}$ has the axioms of $\boldsymbol{S}$ plus the axiom of infinity, and $\boldsymbol{Z} \boldsymbol{F}$ has, in addition, the axiom schema of replacement. ${ }^{(3)}$

We shall need to make frequent use of the following lemma, whose proof is straightforward and will be omitted. Parts (a), (b), and (c) state certain simple invariance properties, while part $(d)$ presents an 'inner' criterion for a supercomplete set to be of the form $R(\lambda)$ for some limit ordinal $\lambda$. Another easily proved fact is that $\vdash R n(x, y) \rightarrow S c(y)$; this will be employed in the sequel without mention.

LEMma 2. (a) $\vdash u \subseteq w \rightarrow[\operatorname{Rel}(u, \varphi) \longleftrightarrow \operatorname{Rel}(w, \operatorname{Rel}(u, \varphi))]$

(b) $\vdash S c(w) \wedge u \in w \rightarrow[\operatorname{Rel}(w, S c(u)) \longleftrightarrow S c(u)]$

(c) $\vdash{ }_{s_{0}} S c(w) \wedge u \in w \rightarrow[\operatorname{Rel}(w, W(u)) \longleftrightarrow W(u)]$

${ }^{2}$ Informally, the sets $R(\alpha)$ are defined by transfinite recursion as follows: $R(\alpha)=$ $\cup\{P(R(\beta)) / \beta<\alpha\}$, where $\cup x$ is the union-set of $x$, and $P(x)$ is the power set of $x$.

${ }^{3}$ For an explicit description of all these axioms and axiom schemata, cf. [9]. The phrase 'the universal closure of' is tacitly understood to precede each description of an axiom given in this paper, so that every axiom is a sentence. 
(d) $\vdash s_{0} W(x) \longleftrightarrow S c(x) \wedge \operatorname{Rel}(x, \rho \wedge(\forall u)(\exists v)(u \in v) \wedge(\exists t)(t=t)),{ }^{(4)}$ where $\rho$ is the sentence $(\forall u)(\exists v, w)(R n(v, w) \wedge u \subseteq w)$.

A very natural theory can be formed by adding $\rho$ to the axioms of $S$ (cf. [7]).

$\S 2$. Principles of reflection. The principle of partial reflection, $P \boldsymbol{R}$, is the axiom schema $\mathcal{P} \rightarrow(\exists u)\left(\operatorname{Sc}(u) \wedge x_{0}, \cdots, x_{n-1} \in u \wedge \operatorname{Rel}(u, \varphi)\right.$. The principle of complete reflection, $\boldsymbol{C R}$, is the schema

$$
(\exists u)\left[\operatorname{Sc}(u) \wedge\left(\forall x_{0}, \cdots, x_{n-1} \in u\right)(\varphi \longleftrightarrow \operatorname{Rel}(u, \varphi))\right] .
$$

In both cases $\varphi$ is supposed to have at most the free variables $x_{0}, \cdots$, $x_{n-1}$. We denote by $\boldsymbol{T}_{0}$ the theory $\boldsymbol{S}_{0}+\boldsymbol{P R} .^{(5)}$

It was shown in [4], Theorem 6, that $\boldsymbol{Z F}=\boldsymbol{S}+\boldsymbol{C R}$. A stronger schema is also valid in $\boldsymbol{Z F}$ (see, e.g., [4]). This is the schema $\boldsymbol{C R}^{*}$ :

$$
(\exists u)\left(t \in u \wedge W(u) \wedge\left(\forall x_{0}, \cdots, x_{n-1} \in u\right)(\rho \longleftrightarrow \operatorname{Rel}(u, \varphi))\right)
$$

(where at most $x_{0}, \cdots, x_{n-1}$ are free in $\varphi$ ). By only slightly modifying the arguments of [4], one sees that, in fact, $\boldsymbol{Z F}=\boldsymbol{S}_{0}+\boldsymbol{C R}{ }^{(6)}$

We shall see below that $\boldsymbol{Z F}$ is much stronger than $\boldsymbol{T}_{0}$. Nonetheless, $\boldsymbol{T}_{0}$ is a very strong set theory. It appears that, roughly speaking, all of the usual constructions of ordinary mathematics, including ordinary set theory, which can be carried out in $\boldsymbol{Z F}$, can also be carried out in $T_{0}$. It seems that only when one turns to notions which are of a metamathematical character can one find propositions valid in $\boldsymbol{Z F}$ but not in $\boldsymbol{T}_{0}$. (Of course such notions can be expressed within set theory itself, so the distinction here is only a vague one). The only way to support these claims would be to prove a great many theorems within $\boldsymbol{T}_{0}$. Perhaps, however, they will be adequately illustrated by the following remarks and examples. ${ }^{(7)}$

4 It was shown in [6] that any supercomplete model of $\boldsymbol{Z F}$ is of the form $R(\lambda)$ for some limit ordinal $\lambda$; references to earlier related results are given there. See also the proof of Theorem 5 in [3]. By defining $R n(x, y)$ in a more awkward way, one can obtain a similar criterion for a supercomplete set to be of the form $R(\alpha)$.

5 The principles $\boldsymbol{C R}$ and $\boldsymbol{P R}$ were introduced and discussed in $[4, \mathbf{5}, \mathbf{3}]$, It may be interesting to note that $\boldsymbol{P R}$ can be put in a form resembling $\boldsymbol{C R}$ even more closely. Indeed, as one easily sees, on the basis of $\mathbf{S}$, the principle $\boldsymbol{P R}$ is equivalent to the schema

$$
(\exists u)\left\{\operatorname{Sc}(u) \wedge y \in u \wedge\left(\forall x_{0}, \cdots, x_{n-1} \in y\right)(\varphi \leftrightarrow \operatorname{Rel}(u, \varphi))\right\},
$$

where at most $x_{0}, \cdots, x_{n-1}$ are free in $\varphi$.

${ }_{6}$ To see this, first note that one easily proves in $\boldsymbol{S}_{0}+\boldsymbol{C R}$ the existence of $0,1,2,3$. Thus one can apply the second argument in the proof of Theorem 2 of [4]. Moreover, by a variant of the same argument one obtains the axiom of pairing. Now all of $\boldsymbol{S}$ and the form of $\boldsymbol{C R}$ used in proving Theorem 6 of [4] follow easily.

7 That the partial reflection principle when added to certain theories $\boldsymbol{T}$ is very strong was already indicated in [3], pp. 164-165 and [5]. However, it was not yet realized there that this applies even to the case when $\boldsymbol{T}$ is $\boldsymbol{S}_{0}$ or $\boldsymbol{S}$. In particular, the question raised in [3], p. 165, whether the existence of $\omega_{1}$ can be established in $\boldsymbol{S}+\boldsymbol{P R}$ is easily answered affirmatively, as we shall see below. 
All the axioms of $S$ follow trivially from $\boldsymbol{T}_{0}$ (to obtain the axiom schema of regularity, one can proceed in the usual way, since, by $\boldsymbol{P R}$, every set is included in a complete set). Since in $S$ one can prove that $x \cup\{x\}$ exists for every $x$, a direct application of $\boldsymbol{P R}$ yields also the axiom of infinity. Thus $\boldsymbol{Z} \subseteq \boldsymbol{T}_{0}$.

We shall briefly sketch a proof in $T_{0}$ of the proposition $\rho$ (of Lemma 2): If $\rho$ does not hold, then by regularity there is a $u$ such that

$$
\sim(\exists x, y)(\operatorname{Rn}(x, y) \wedge u \subseteq y)
$$

while

$$
\left(\forall u^{\prime} \in u\right)\left(\exists x^{\prime}, y^{\prime}\right)\left(R n\left(x^{\prime}, y^{\prime}\right) \wedge u^{\prime} \leqq y^{\prime}\right) .
$$

By $\boldsymbol{P R}, u$ belongs to some supercomplete set $t$ such that

$$
\left(\forall u^{\prime} \in u\right)\left(\exists x^{\prime}, y^{\prime} \in t\right) \operatorname{Rel}\left(t, \operatorname{Rn}\left(x^{\prime}, y^{\prime}\right) \wedge u^{\prime} \subseteq y^{\prime}\right) .
$$

It follows, as one may check by looking at Definition 1, that

$$
\left(\forall u^{\prime} \in u\right)\left(\exists x^{\prime}, y^{\prime} \in t\right)\left(R n\left(x^{\prime}, y^{\prime}\right) \wedge u^{\prime} \subseteq y^{\prime}\right) .
$$

Now on the basis of $\boldsymbol{Z}$ there exist $z, x$, and $y$ such that, for any $x^{\prime}, y^{\prime}$

$$
\begin{aligned}
& R l\left(x^{\prime}, y^{\prime}, z\right) \longleftrightarrow x^{\prime}, y^{\prime} \in t \wedge R n\left(x^{\prime}, y^{\prime}\right), \\
& x^{\prime} \in x \longleftrightarrow\left(\exists y^{\prime}\right)\left(x^{\prime}, y^{\prime} \in t \wedge R n\left(x^{\prime}, y^{\prime}\right)\right), \text { and } \\
& u^{\prime} \in y \longleftrightarrow\left(\exists x^{\prime}, y^{\prime}\right)\left(x^{\prime}, y^{\prime} \in t \wedge R n\left(x^{\prime}, y^{\prime}\right) \wedge u^{\prime} \subseteq y^{\prime}\right) .
\end{aligned}
$$

Again on the basis of $Z$, it is a simple exercise to infer that $R n(x, y)$, $z$ being the set whose existence is required in Definition 1 . Since, by (2) and (3), $u \subseteq y$, we have obtained a contradiction.

Let $\boldsymbol{P} \boldsymbol{R}^{*}$ be the schema whose description has ' $W(u)$ ' when that of $\boldsymbol{P R}$ has ' $S c(u)$ '. Since $\rho$ is valid in $\boldsymbol{T}_{0}$, we see by Lemma $2(d)$ that $\boldsymbol{P} \boldsymbol{R}^{*}$ is valid in $T_{0}$.

One can prove in $\boldsymbol{T}_{0}$ the following theorem: For any set $x$ well ordered by a relation $y$, there exists an ordinal $\alpha$ such that $\left\langle\alpha, \epsilon_{\alpha}\right\rangle$ is isomorphic to $\langle x, y\rangle$. (By $\varepsilon_{z}$ we mean $\{\langle u, v\rangle / u, v \in z \wedge u \in v\}$.) This theorem is proved by transfinite induction within $\langle x, y\rangle$, invoking $\boldsymbol{P R}$ at the limit stages.

One now easily proves by induction on $\alpha$, in $\boldsymbol{T}_{0}$, that the initial ordinals $\omega_{\alpha}$ exist for every $\alpha$, again invoking $\boldsymbol{P R}$ when $\alpha$ is a limit ordinal.

We now construct a second theory, $\boldsymbol{T}_{1}$, as follows. The theory $\boldsymbol{T}_{1}$, has in addition to $\varepsilon$, the individual constant $V$. Its axioms are those of $\boldsymbol{S}+\rho$ together with $S c(V)$ and the following axiom schema, $\boldsymbol{P} \boldsymbol{R}_{1}$ :

$$
\begin{gathered}
x_{0}, \cdots, x_{n-1} \in V \wedge \psi \rightarrow(\exists u \in V)\left[\operatorname{Sc}(u) \wedge x_{0}, \cdots, x_{n-1} \in u \wedge\right. \\
\operatorname{Rel}(u, \psi)]
\end{gathered}
$$


(where the free variables of $\psi$ are among $\left.x_{0}, \cdots, x_{n-1}\right){ }^{(8)}$ As before, by Lemma 2(d), the schema $\boldsymbol{P R}_{1}^{*}$ having ' $W(u)$ ' in place of ' $S c(u)$ ' in (4), is also valid in $T_{1}$.

If $\sigma$ is any sentence valid in $\boldsymbol{T}_{0}$, then $\operatorname{Rel}(V, \sigma)$ is valid in $\boldsymbol{T}_{1}$ (i.e., $\boldsymbol{T}_{0} \subseteq \boldsymbol{T}_{1} / V$ ). Indeed, suppose $\sigma$ is (the universal closure of) an instance of $\boldsymbol{P R}$ applied to the formula $\varphi$. Then $\operatorname{Rel}(V, \sigma)$ may be derived in $\boldsymbol{T}_{1}$ by applying $\boldsymbol{P R}_{1}$ with $(\exists w)\left(S c(w) \wedge x_{0}, \cdots, x_{n-1} \in w \wedge \operatorname{Rel}(w, \varphi)\right)$ for $\psi$ (and noting Lemma 2(a)).

§3. Formalized syntactical and semantical notions. As is well known we can, if we wish, conceive of the expressions of $\boldsymbol{T}_{1}$ as natural numbers, of the concatenation of expressions as some specific elementary number-theoretical operation, etc. Let us assume henceforth that this has been done explicitly in some standard way. It follows that we can get counterparts of metamathematical discussions such as those in $\S 1$ and $\S 2$ within the theory $S$ by 'translating' those considerations to $S$. In this way we arrive at several special formulas in (addition to those in Definition 1) which will be needed. In Definition 3, below, we do not exhibit these formulas explicitly, but only state a version of the intended explicit formula in English. The explicit formula is to be obtained from the English version by understanding notions defined in $\S 1$ or $\S 2$ in the way specified there, understanding such terms as 'function', 'natural number', etc., which have not been defined here, in the standard way, and then translating in an intensional way. ${ }^{(9)}$

Definition 3. (In the following, $\boldsymbol{T}$ is to be $\boldsymbol{T}_{0}, \boldsymbol{T}_{1}, \boldsymbol{T}_{1} / V, T_{0}+\sigma$, $T_{1}+\Sigma$, or $T_{1} / V+\sigma$.)

(a) $A x_{T}(x)$ :

(b) Con $_{T}$ :

(c) $A s(x, y, z)$ : $x$ is an axiom of $\boldsymbol{T}^{\left({ }^{(10)}\right.}$

No contradiction is derivable from the axioms of $T$.

$S c(z), z \neq 0, y$ is a formula of $\boldsymbol{T}_{0}$, and $x$ is a function on the set of free variables of $y$ assigning to each a member of $z$.

8 The possibility of forming 'higher order' partial reflection principles, like $\boldsymbol{P R}_{1}$, is already suggested in [3], p. 165.

9 The only rigorous procedure would be to describe these formulas explicitly; but the present procedure, which saves much space, seems unlikely to cause confusion.

10 The formulas $A x_{T}(x)$ should (and obviously can) be constructed so as to meet the requirements of Feferman and Montague (cf. [2]). Though the notion ' $\boldsymbol{T}_{0}$ ', for example, has no representation in $\boldsymbol{S}$, the notion 'axiom of $\boldsymbol{T}_{0}$ ' obviously does. When referring to the definition of $\boldsymbol{P} \boldsymbol{R}_{1}$ in $\S 2$ in constructing the formulas $A x_{\boldsymbol{T}}(x)$, one should consider that $\boldsymbol{P} \boldsymbol{R}_{1}$ is the set of all sentences (4) where $x_{0}, \cdots, x_{n-1}, u$ are arbitrary (rather than particular) distinct variables; and similarly for $\boldsymbol{P R}$. 
(d) $\operatorname{St}(x, y, z): \quad A s(x, y, z)$ and $x$ satisfies $y$ in $\left\langle z, \varepsilon_{z}\right\rangle$. .11) $^{\text {. }}$

(d') $S t^{\prime}(x, y, z, u): S c(z), u \in z, y$ is a formula of $\boldsymbol{T}_{1}, x$ is a function on the set of free variables of $y$ to $z$, and $x$ satisfies $y$ in $\left\langle z, \varepsilon_{z}, u\right\rangle$. ${ }^{(12)}$

(e) $\operatorname{Mod}_{T_{0}}(x)$ : For any $w$, if $A x_{T_{0}}(w)$ then $S t(0, w, x)$.

(f) $\operatorname{Neg}(x, y): \quad x$ is a formula and $y$ is the negation of $x$.

(g) Relat $(x, y, z): y$ is a formula, $x$ is a variable not free in $y$, and $z$ is the result of relativizing the quantifiers in $y$ to $x$.

We denote by $\boldsymbol{S}^{\prime}$ the theory whose non-logical symbols are $\varepsilon, 0$ and $S$ and whose axioms are those of $S$ plus $(\forall x)(x \notin 0)$ and

$$
(\forall y)(y \in S x \longleftrightarrow y \in x \vee y=x) .
$$

Obviously, a formula of $S$ is valid in $S^{\prime}$ if and only if it is valid in $S$. The terms $\overline{0}, \overline{1}, \cdots$ of $S^{\prime}$ are defined recursively by the requirements: $\overline{0}$ is 0 and $\overline{n+1}$ is $S(\bar{n})$.

The following lemma is easily established. (In reading it, recall that every expression of $\boldsymbol{T}_{1}$ is a natural number).

\section{LEMMA 4.}

(a) $\vdash_{S} A s(s, t, u) \wedge N e g\left(t, t^{\prime}\right) \rightarrow\left[S t\left(s, t^{\prime}, u\right) \longleftrightarrow \sim S t(s, t, u)\right]$

and the analogous conditions concerning atomic formulas, joint denial, etc., in place of negation, or $S t^{\prime}$ in place of $S t$.

(b) Suppose the free variables of $\phi$ are $v_{k_{0}}, \cdots, v_{k_{m}-1}$, where

$$
\begin{aligned}
k_{0} & <k_{1}<\cdots<k_{m-1}<n . \quad \text { Then } \\
\vdash{ }_{s} A s(s, \bar{\varphi}, u) & \wedge R l\left(\bar{v}_{k_{0}}, z_{k_{0}}, s\right) \wedge \cdots \wedge \operatorname{Rl}\left(\bar{v}_{k_{m-1}}, z_{k_{m-1}}, s\right)
\end{aligned}
$$$$
\rightarrow\left[\operatorname{St}(s, \bar{\varphi}, u) \longleftrightarrow \operatorname{Rel}\left(u, \varphi\left(z_{0}, \cdots, z_{n-1}\right)\right)\right] .
$$

(c) $\vdash{ }_{S} S c(w) \wedge u \in w \wedge A s(s, t, u) \wedge \operatorname{Relat}\left(x, t, t^{\prime}\right)$

$$
\begin{aligned}
& \wedge(\forall z)\left(z \in s^{\prime} \longleftrightarrow z \in s \vee O c(x, u, z)\right) \\
& \quad \rightarrow\left[S t(s, t, u) \longleftrightarrow \operatorname{St}\left(s^{\prime}, t^{\prime}, w\right)\right] .
\end{aligned}
$$

11 The second clause may be understood (still in English) as follows: There exists a set $w$ such that (i) for any $f, t<f, t\rangle \in w$ if and only if $f$ is a function on the set of variables (free or bound) of $y$ to $z, t$ is a subformula of $y$, and there exist variables $\xi, \eta$ and formulas $t^{\prime}, t^{\prime \prime}$ such that either (a) $t$ is the formula $\xi=\eta$ and $f(\xi)=f(\eta)$, (b) $t$ is the formula $\xi \in \eta$ and $f(\xi) \in f(\eta)$, (c) $t$ is the formula $t^{\prime} \mid t^{\prime \prime}$ and $\left\langle f, t^{\prime}\right\rangle \notin w$ and $\left\langle f, t^{\prime \prime}\right\rangle \notin w$, or (d) $t$ is the formula $(\xi \xi) t^{\prime}$ and there is a $v$ such that

$$
<(f-\{<\xi, f(\xi)>\}) \cup\{<\xi, v>\}, t^{\prime}>\in w,
$$

and (ii) for some $x^{\prime},\left\langle x^{\prime}, y\right\rangle \in w$ and $x \subseteq x^{\prime}$.

12 We shall understand the last clause as follows: For any variable $\xi$ not occurring in $y$, if $y^{\prime}$ is the result of substituting $\xi$ for $V$ throughout $y$ and $x^{\prime}=x \cup\{\langle\xi, u\rangle\}$, then St $\left(x^{\prime}, y^{\prime}, z\right)$. 
(d) $\vdash_{s} W(v) \wedge s, t, u \in v \rightarrow[\operatorname{Rel}(v, S t(s, t, u)) \longleftrightarrow S t(s, t, u)]$.

LEMMA 5. $\vdash_{s} W(w) \wedge W(v) \wedge v \in w \rightarrow$

$$
\left\{\operatorname{Mod}_{{ }^{\prime}}(w, v) \longleftrightarrow(\forall s, t \in v)[S t(s, t, w) \rightarrow(\exists u \in v) \operatorname{St}(s, t, u)]\right\} .
$$

Proof. Under this hypothesis we have $\operatorname{Mod}_{S}(w)$, as is well known. One easily checks that $S t^{\prime}(0, \overline{S c(v),} w, v)$. That $\operatorname{St}(0, \bar{\rho}, w)$ is immediate from Lemmas 4(a) and 2(d). It follows that $\operatorname{Mod}_{T_{1}}^{\prime}(w, v)$ if and only if each instance of $\boldsymbol{P R}_{1}$, is satisfied by 0 in $\left\langle w, \varepsilon_{w}, v\right\rangle$. Obviously, since $W(v)$, any finite sequence of members of $v$ belongs to $v$ and $\omega \leqq v$. Now, by looking at (4) and applying Lemmas 4(a), 4(b), 2(b), and 4(c), we see at once that the desired conclusion holds.

Let $\boldsymbol{T}$ be a set theory involving only $\varepsilon . \quad T$ is called essentially reflexive over $\boldsymbol{T}_{0}$ if $\boldsymbol{S} \subseteq \boldsymbol{T}$ and, for every $\sigma, \vdash_{\boldsymbol{T}} \boldsymbol{\sigma} \rightarrow$ Con $_{\boldsymbol{T}_{0}+\sigma}$. An instance of a general observation due to Montague is the following fact: If $\boldsymbol{T}$ is essentially reflexive over $\boldsymbol{T}_{0}$ then $\boldsymbol{T}$ is an essentially infinite extension of $\boldsymbol{T}_{0}$, i.e., $\boldsymbol{T}$ is an extension of $\boldsymbol{T}_{0}$ and no consistent extension of $\boldsymbol{T}$ without new symbols can be obtained by adding a finite number of axioms to those of $\boldsymbol{T}_{0}$. These statements remain correct if ' $\boldsymbol{T}_{0}$ ' is replaced everywhere by ' $\boldsymbol{T}_{1} / V$ '. ${ }^{(13)}$

\section{$\S 4$. Models of $T_{0}$ and $T_{1}$ within $Z F$.}

Definition 6. $Q(x)$ for $W(x) \wedge(\forall w)(\forall s, t \in x)[(S t(s, t, w) \rightarrow$

$$
(\exists u \in x) \operatorname{St}(s, t, u)]
$$

(' $Q(x)$ ' may be read ' $x$ is a $Q$-set'.)

THEOREM 7. (a) $\vdash_{z F}(\exists v)(t \in v \wedge Q(v))$.

(b) $\vdash{ }_{z F} \sigma \rightarrow(\exists v)(t \in v \wedge \operatorname{Rel}(v, \sigma) \wedge Q(v))$.

(In view of Lemma 5 and Definition 6, Theorem 7(a) implies that one can prove in $\boldsymbol{Z F}$ that statement: there exist arbitrarily large limit ordinals $\lambda$ such that for every limit ordinal $\lambda^{\prime}>\lambda, \quad<R\left(\lambda^{\prime}\right), \varepsilon_{R\left(\lambda^{\prime}\right)}, R(\lambda)$ $>$ is a model of $T_{1}$.)

Proof. By (1), taking $(\exists w) S t(s, t, w)$ for $\varphi$, we obtain $(\exists v)\{t \in v \wedge W(v) \wedge(\forall s, t \in v)[(\exists w) \operatorname{St}(s, t, w) \longleftrightarrow \operatorname{Rel}(v,(\exists u) \operatorname{St}(s, t, u))]\}$. Hence, by Lemma 4(d),

$(\exists v)\{t \in v \wedge W(v) \wedge(\forall s, t \in v)(\forall w)[S t(s, t, w) \rightarrow(\exists u \in v) \operatorname{St}(s, t, u)]\} ;$ and by Definition 6 this is what was to be proved in (a). By taking,

${ }^{13}$ It is here that we are relying on the fact that the formulas $A x_{T_{0}}(x)$ and $A x_{T_{1} v}(x)$ can be constructed in a special way. Cf. [2]. 
instead, $\sigma \wedge(\exists w) S t(s, t, w)$ for $\varphi$ in (1), one obtains in the same way (b).

Corollary 8. $\quad \boldsymbol{Z} \boldsymbol{F}$ is essentially reflexive over $\boldsymbol{T}_{1} / V$ and over $\boldsymbol{T}_{0}$.

Proof. From Theorem 7(b) and Lemma 5 (as well as Lemmas 4(b) and 2(a)) it obviously follows that

$$
\vdash_{z \boldsymbol{F}} \boldsymbol{\sigma} \rightarrow(\mathbf{3} v, w) \operatorname{Mod}_{T_{1}+\operatorname{Rel}(V, \sigma)}^{\prime}(w, v) .
$$

It can be proved in the standard way that

$$
\vdash{ }_{S} \operatorname{Mod}_{T_{1}+\Sigma}(w, v) \rightarrow \operatorname{Con}_{T_{1}+\Sigma}
$$

and obviously

$$
\vdash{ }_{S} \operatorname{Con}_{T_{1}+R e l(V, \sigma)} \rightarrow \operatorname{Con}_{T_{1} / V+\sigma} .
$$

Hence $\vdash_{z F} \sigma \rightarrow C o n_{T_{1}+\sigma}$. Since by $\S 2$, one can prove within $S$ that $\boldsymbol{T}_{0} \subseteq \boldsymbol{T}_{1} / V$, it follows that also $\vdash_{z \boldsymbol{F}} \sigma \rightarrow \operatorname{Con}_{\boldsymbol{T}_{0}+\sigma}$, which completes the proof.

Definition 9. By $Q^{\prime}(x, y)$ we shall mean the formula

$$
\begin{gathered}
\operatorname{Ord}(x) \wedge(\exists z)\{(\forall u)(u \in x \rightarrow(\exists v) R l(u, v, z)) \wedge(\forall u, v)[(u \in x \wedge \operatorname{Rl}(u, v, z)) \\
\vee(u=x \wedge v=y) \rightarrow Q(v) \wedge\left(\forall u^{\prime}, v^{\prime}\right)\left(u^{\prime} \in u \wedge \operatorname{Rl}\left(u^{\prime}, v^{\prime}, z\right) \rightarrow v^{\prime} \in v\right) \\
\wedge\left(\forall v^{\prime \prime}\right)\left[Q\left(v^{\prime \prime}\right) \wedge\left(\forall u^{\prime}, v^{\prime}\right)\left(u^{\prime} \in u \wedge \operatorname{Rel}\left(u^{\prime}, v^{\prime}, z\right) \rightarrow v^{\prime} \subseteq v^{\prime \prime} \wedge v^{\prime} \neq v^{\prime \prime}\right)\right. \\
\left.\left.\left.\rightarrow v \subseteq v^{\prime \prime}\right]\right]\right\} .
\end{gathered}
$$

(Thus ' $Q$ ' $(x, y)$ ' may be read ' $x$ is an ordinal and the $x$ th $Q$-set exists and equals $y$ '.)

From Theorem 7(a) it obviously follows that

THEOREM 10. $\vdash_{z F}(\forall t)\left(\operatorname{Ord}(t) \rightarrow(\exists u) Q^{\prime}(t, u)\right)$.

$\S 5$. Models of $\boldsymbol{T}_{0}$ within $\boldsymbol{T}_{1}$. We shall now see that the consistency of $\boldsymbol{T}_{0}$ can be established, by a different method, within a theory much weaker than $\boldsymbol{Z F}$.

THEOREM 11. $\vdash{ }_{T_{1}} Q(V)$.

Proof. By Lemma 2(d) and the discussion in $\S 2$, we see that $W(V)$. Thus, by Definition 6, what we must show is that

$$
(\forall w)(\forall s, t \in V)[S t,(s, t, w) \rightarrow(\exists u \in V) S t(s, t, u)] .
$$

Suppose $s, t \in V$ and $S t(s, t, w)$. Then obviously $(\exists u) S t(s, t, u)$, so that by $\boldsymbol{P} \boldsymbol{R}_{1}^{*}$ we have: 


$$
(\exists z \in V)[W(z) \wedge s, t \in z \wedge \operatorname{Rel}(z,(\exists u) \operatorname{St}(s, t, u))] .
$$

Since $S c(V)$, it follows at once by Lemma $4(\mathrm{~d})$ that $(\exists u \in V) S t(s, t, u)$, as was to be proved.

CoRollary 12. $\boldsymbol{T}_{1} / V$ is essentially reflexive over $\boldsymbol{T}_{0}$.

Proof. In exactly the same way that Lemma 5 was proved, one sees that

$$
\vdash_{S} W(v) \rightarrow\left[\operatorname{Mod}_{T_{0}}(v) \longleftrightarrow(\forall x, y \in v)[S t(x, y, v) \rightarrow(\exists u \in v) \operatorname{St}(x, y, u)] .\right.
$$

Now the corollary follows easily from Theorem 11; we omit the details.

By slightly modifying the proof of Corollary 12 (and, of course various lemmas and theorems used in its proof) one can verify that Corollary 12 remains valid if $\boldsymbol{T}_{1}$ is replaced by the weaker theory

$$
\boldsymbol{S}_{0}+\operatorname{Sc}(V)+\boldsymbol{P R}_{1} \text {. }
$$

If the latter theory is consistent, it remains so, as one easily checks, on the addition of the axiom $x \leqq V$, which converts it into a Bernaystype set theory.)

§6. Ackermann's set theory. A new system of set theory was proposed by Ackermann in [1]. A further study and development of it was made in [3]. The theory $\boldsymbol{A}$ of [3] has the non-logical constants $\varepsilon$ and $V$. Its axioms and axiom schemata are

( $\alpha) \quad(\exists x)(\forall y)(y \in x \longleftrightarrow y \in V \wedge \Phi) \quad(x$ not free in $\Phi)$

(B) Extensionality

(y) $x_{0}, \cdots, x_{n-1} \in V \wedge(\forall y)(y \in x \longleftrightarrow \chi) \wedge x \subseteq V \rightarrow x \in v$ (where the free variables of $\chi$ are among $y, x_{0}, \cdots, x_{n-1}$ )

(ס) $S c(V)$.

(Actually, our axiom $(\alpha)$ differs superficially from that in [3], but is obviously logically equivalent to it. Our axiom $(\boldsymbol{\gamma})$ also differs from that in [3], but it is clear that, on the basis of $(\alpha)$ and $(\beta)$, the two are equivalent. The theory $\boldsymbol{A}$ of [3] only differs, in turn, from the original theory of Ackermann [1] in having an individual constant $V$ in place of a singularly predicate $M$.)

We denote by $\boldsymbol{A}^{*}$ the theory $\boldsymbol{A}+\operatorname{Rel}(V, \chi)$, where $\chi$ is the axiom of regularity.

Ackermann established in [1] that all the axioms of $\boldsymbol{Z}$ with the exception of the regularity schema are valid in $\boldsymbol{A} / V$. In [3] various further results of this kind were established, which we shall need to use. The following Lemma 13 lists a number of these or their easy consequences. It is necessary here to correct an error in [3]. Lemma 3 there which is the same as Lemma 13(a) below) does not appear to be correct, as it stands. However, if the definition of $\operatorname{Ord}(\alpha)$ 
in [3] is replaced by that adopted here (in Definition 1), then the proof sketched in [3] for Lemma 3 can indeed be carried out, as one easily checks; moreover, everything following Lemma 3 in [3] remains valid. Hencerforth, in referring to [3] we have in mind that this change in [3] has been carried out.

LEMMA 13.

(a) $\vdash{ }_{A} \operatorname{Ord}(x) \wedge \operatorname{Ord}(y) \wedge x \in V \rightarrow x \in y \vee x=y \vee y \in x$

(b) $\vdash{ }_{A} x \in V \vee y \in V \rightarrow[R n(x, y) \longleftrightarrow x \in V \wedge y \in V$ $\wedge \operatorname{Rel}(V, \operatorname{Rn}(x, y))]$

(c) $\vdash_{A / V}(\forall y \in x)(\exists u, v)(R n(u, v) \wedge y \in v) \rightarrow(\exists u, v)(R n(u, v) \wedge x \in v)$

(d) $\vdash{ }_{A} y \in V \rightarrow[W(y) \longleftrightarrow \operatorname{Rel}(v, W(y))]$

(e) $\vdash{ }_{A^{*}} W(V)$

(f) $\vdash{ }_{A} R n(x, y) \wedge R n\left(x^{\prime}, y^{\prime}\right) \wedge x \in V \wedge x \subseteq x^{\prime} \rightarrow y \subseteq y^{\prime}$.

Proof. Since our definition of $R n(x, y)$ differs a bit from that in [3], most of these results cannot be derived directly from what is in [3]. Rather, we must first change slightly the arguments in [3] so as to obtain the same results for our $R n(x, y)$ as are established in [3] for $R n(x, y)$ (i.e., $y=\Re(x))$ there; this is easily done. With this understanding (f) can easily be established from what is in [3]. (b) follows directly from what is in [3] plus the fact that $\vdash_{A} R n(x, y) \wedge y \in V \rightarrow x \in V$. The latter may be established as follows: Suppose that $R n(x, y) \wedge y \in V \wedge$ $x \notin V$. It follows at once by Definition 1 that $(\forall u \in x)(\exists v) R n(u, v)$, and hence by (a) that $(\forall u \in V)(\operatorname{Ord}(u) \rightarrow(\exists v) R n(u, v))$. By (a) and (f),

$$
(\forall u \in V)(\forall v)(R n(u, v) \rightarrow v \subseteq y) .
$$

One easily establishes that $(\forall u \in V)(\forall v)(R n(u, v) \rightarrow u \subseteq v$ ) (by at least ordinal argument based on $(\alpha)$ and Definition 1$)$. Therefore

$$
(\forall u \in V)(\operatorname{Ord}(u) \rightarrow u \subseteq y),
$$

and hence, by $(\alpha)$ and $(\delta)$, there is a $z \in V$ such that

$$
\forall u(u \in z \longleftrightarrow u \in V \wedge \operatorname{Ord}(u)) .
$$

Now, using Definition 1 and (a), one verifies that $\operatorname{Ord}(z)$ and $z \in z$, a contradiction.

(c) is Corollary 4(a) of [3]. (d) is an immediate consequence of (b). Finally, (e) can be derived at once from Theorem 5 of [3] by taking $\sim W(x)$ for $\phi(x)$.

We now wish to present two results concerning the relativized axiom of regularity. The first is that the axiom schema of regularity is valid in $\boldsymbol{A}^{*} / V$ (so that our $\boldsymbol{A}^{*}$ coincides with that of [3] and we have $\boldsymbol{Z} \subseteq \boldsymbol{A}^{*} / V$ ). In order to prove this it suffices, as is well-known, to show that, on the 
basis of $\boldsymbol{A}$, every set $x$ is included in a complete set. To see this, one notes that the intersection of all complete classes including $x$, of which $V$ is one, exists (by $(\alpha)$ ) and is such a set (because of $(\boldsymbol{\gamma})$ ). The second result is that the situation for $\boldsymbol{A}$ with respect to regularity is like that which von Neumann showed to obtain for the usual set theories, namely:

Theorem 14. If $\boldsymbol{A}$ is consistent, so is $\boldsymbol{A}^{*}$. Indeed, $\boldsymbol{A}^{*}$ is interpretable within $\boldsymbol{A}$ (in the sense of [8]). ${ }^{(14)}$

Proof. As in [3], we note that by axiom $(\boldsymbol{\alpha})$ it is provable in $\boldsymbol{A}$ that there exists a unique class $\pi$ such that

$$
(\forall x)[x \in \pi \longleftrightarrow x \in V \wedge \operatorname{Rel}(V,(\exists u, v)(\operatorname{Rn}(u, v) \wedge x \in v))] .
$$

Let $A^{\prime}$ be obtained from $\boldsymbol{A}$ by adding the individual constant $\pi$ and the axiom just displayed.

We will show that each axiom of $\boldsymbol{A}$ is valid in $\boldsymbol{A}^{\prime}$ if in it $V$ is replaced by $\pi$. This is immediate for axiom $(\alpha)$, and also for $(\beta)$, since $\pi \subseteq V$, and is easily proved for $(\delta)$. Finally, that it applies to $(\boldsymbol{\gamma})$ is an immediate consequence of $(\gamma)$ itself and Lemma 13(c) above, which says exactly that $x \in V \wedge x \subseteq \pi \rightarrow x \in \pi$.

To complete the proof it clearly suffices to show that the axiom of regularity relativized to $\pi$ is valid in $\boldsymbol{A}^{\prime}$. But this can be done by employing the usual argument (involving the notion of the smallest $\boldsymbol{\alpha}$ such that $x \subseteq R(\alpha)$ ).

In view of Theorem 14 (and the details in its proof) we shall henceforth deal exclusively with the system $\boldsymbol{A}^{*}$. It was shown in [3] that $\boldsymbol{A}^{*} / V \subseteq \boldsymbol{Z F}$. As was mentioned in the introduction, it is an open problem whether $\boldsymbol{Z F}$ coincides with $\boldsymbol{A}^{*} / V$. It was also established in [3] that whenever $\sigma$ is valid in $\boldsymbol{A}^{*}$, then it is valid in $\boldsymbol{Z F}$. As we might conjecture from looking at the axioms of $\boldsymbol{A}^{*}$, the converse here is false; this has recently been proved by Grewe and Vaught.

In [3] (Theorem 5,) it was proved that a new, strong type of partial reflection principle is valid in $\boldsymbol{A}^{*}$. This is the following schema, which we shall call $\boldsymbol{R}:^{(15)}$

$$
x_{0} \cdots, x_{n-1} \in V \wedge \varphi(V) \rightarrow(\exists u \in V) \varphi(u)
$$

(where at most $v_{0}, x_{0}, \cdots, x_{n-1}$ are free in $\varphi$ ).

14 A closely related observation was made in [3], p. 163. What is there can be used together with our observation below that $(\gamma)$ can be replaced by $\boldsymbol{R}$ to give a slightly different proof of Theorem 14.

It may be mentioned that the second author has established a stronger result: The theory obtained by adding to $\boldsymbol{A}$ the unrelativized axiom of regularity is relatively interpretable within $\boldsymbol{A}$.

${ }_{15}$ The schema appearing in $[\mathbf{3}]$, Theorem 5 , is actually somewhat stronger (formally). 
It may be remarked that, in fact: In the axioms of $\boldsymbol{A}^{*},(\boldsymbol{\gamma})$ may be replaced by $\boldsymbol{R}$. To see this, suppose that $(\alpha),(\beta), \boldsymbol{R},(\delta)$, the axiom of regularity relativized to $V$, and the hypothesis of the typical instance of $(\boldsymbol{\gamma})$ all hold. Then we have: There exists $x \subseteq V$ such that for any $y, y \in x$ if and only if $\chi$. Hence, by $\boldsymbol{R}$, for some $u \in V$, there exists $x^{\prime} \subseteq u$ such that, for any $y, y \in x^{\prime}$ if and only if $\chi$. By $(\beta), x=x^{\prime}$, and, by $(\delta), x^{\prime} \in V$, so that $x \in V$, as was to be proved.

As was observed in [3], from the validity in $\boldsymbol{A}^{*}$ of $\boldsymbol{R}$ it follows easily that $\boldsymbol{P R}$ is valid in $\boldsymbol{A}^{*} / V$, so that $\boldsymbol{T}_{0} \subseteq \boldsymbol{A}^{*} / V$. It was briefly indicated there that $\boldsymbol{R}$ implies as well various 'higher order' reflection principles, and that $\boldsymbol{R}$ may be used to establish the existence of various proper classes. ${ }^{(16)}$

We shall now establish a series of results whose end product will be the proof that the formulas shown in Theorem 7 and 10 to be valid in $\boldsymbol{Z F}$ are also valid in $\boldsymbol{A}^{*}$. These results constitute a further general development of the theory of Ackermann, which includes and extends the ideas mentioned in [3] concerning higher order reflection principles. An interesting feature will be the proof (on the basis of $\boldsymbol{A}^{*}$ ) of the existence of well-behaved classes much larger than $V$ itself.

From now on ' $\mathcal{}$ ' and ' $\mathcal{P}$ ' will always denote formulas with at most the free variables $v_{0}$ and $v_{1}$. Let $S_{\varphi}(x, y, z)$ be the formula

$$
z \in y \wedge \mathcal{P}(x, y) \wedge W(y) \wedge\left(\forall y^{\prime}\right)\left(z \in y^{\prime} \wedge \varphi\left(x, y^{\prime}\right) \wedge W\left(y^{\prime}\right) \rightarrow y \subseteq y^{\prime}\right) .
$$

From the axiom $(\boldsymbol{\beta})$ of extensionality it obviously follows that

$$
\vdash{ }_{A} * S_{\varphi}(x, y, z) \wedge S_{\varphi}\left(x, y^{\prime}, z\right) \rightarrow y=y^{\prime} .
$$

LemMa 15. $\vdash_{A^{*}} x, z \in V \wedge \rho(x, V) \rightarrow(\exists y \in V) S_{\varphi}(x, y, z)$.

Proof. Assume $x, z \in V$ and $\mathcal{P}(x, V)$. Then, by Lemma 13(e) and the principle $\boldsymbol{R}$, we have

$$
(\exists y \in V)(z \in y \wedge \varphi(x, y) \wedge W(y)) .
$$

That, as is to be proved, there is a smallest such $y$ now follows easily from (6) by means of Lemma 13(a), (b), (f).

THEOREM 16. $\vdash_{A^{*}} x \in V \wedge \mathcal{P}(x, V) \rightarrow(\exists y) S_{\varphi}(x, y, V)$.

Proof. Suppose that $x \in V \wedge \varphi(x, V) \wedge \sim(\exists y) S_{\varphi}(x, y, V)$. Then by $\boldsymbol{R},(\exists z \in V) \sim(\exists y) S_{\varphi}(x, y, z)$, which contradicts Lemma 15.

If one ignores the part of its conclusion saying that $y$ is smallest, Theorem 16 may be regarded as an inverted form of the principle $\boldsymbol{R}$.

We shall denote by $T d_{\varphi}(x, y)$ the formula

16 Cf. [3], pp. 162-165. 


$$
x \in V \wedge \varphi(x, V) \wedge S_{\varphi}(x, y, V),
$$

which will be read ' $y$ is $\varphi$-tied to $V$ by $x$ '. Thus Theorem 16 says that if $x \in V$ and $\phi(x, V)$ then there exists a class which is $\phi$-tied to $V$ by $x$.

In some applications of Theorem 16 it is suggestive to have in mind the following 'metamathematical principle', which follows at once from it (by taking $\sim \varphi^{\prime}$ for $\varphi$ in Theorem 16):

If $y$ is not free in $\psi$ and if, for every $\varphi$

$$
\vdash_{A^{*}} \psi^{\prime} \rightarrow\left[T d_{\varphi}(x, y) \rightarrow \phi^{\prime}(x, y)\right] \text { then } \vdash_{A^{*}} \psi_{\boldsymbol{r}} \rightarrow\left(x \in V \rightarrow \phi^{\prime}(x, V)\right) \text {. }
$$

(That is, in $\boldsymbol{A}^{*}$, for a given set $x$, if we want to show that $\varphi^{\prime}(x, V)$, it is enough to show that $\varphi^{\prime}(x, y)$ for every $y$ tied to $V$ by $\left.x\right)$.

$$
\begin{gathered}
\text { TheOREM 17. } \vdash{ }^{*} T d_{\varphi}(x, y) \wedge x_{0}, \cdots, x_{n-1} \in V \wedge \psi(y) \\
\rightarrow\left(\exists y^{\prime} \in V\right) \psi\left(y^{\prime}\right)
\end{gathered}
$$

(where \& has no free variables except $v_{0}, x_{0}, \cdots, x_{n-1}$ ).

Proof. Our hypothesis is that

$$
x \in V \wedge \varphi(x, V) \wedge S_{\varphi}(x, y, V) \wedge x_{0}, \cdots, x_{n-1} \in V \wedge \psi(y) .
$$

It follows, by (5) that $\left(\forall y^{\prime}\right)\left(S_{\varphi}\left(x, y^{\prime}, V\right) \rightarrow \psi\left(y^{\prime}\right)\right)$. Hence, by $\boldsymbol{R}$, there is a set $z$ such that $x_{0}, \cdots, x_{n-1} \in z \wedge\left(\forall y^{\prime}\right)\left(S_{\varphi}\left(x, y^{\prime}, z\right) \rightarrow \psi\left(y^{\prime}\right)\right)$. Since by Lemma $15\left(\exists y^{\prime} \in V\right) S_{\varphi}\left(x, y^{\prime}, z\right)$, we conclude that there is a set $y^{\prime}$ such that $\psi\left(y^{\prime}\right)$.

The schema in Theorem 17 is similar to $R$ itself. Roughly speaking, $\boldsymbol{R}$ says that if $V$ stands in a given (expressible) relation to some fixed sets, then there is a set $v$ which stands in the same relation to the fixed sets. Similarly, the schema of Theorem 17 says that if a class $y$ is tied to $V$ then, if $y$ is in a given relation to some fixed sets, then there exists a set $y^{\prime}$ which is in the same relation to the same sets. Since much can be proved about arbitrary sets, it follows that much can be proved concerning classes tied to $V$; (this will be illustrated in the first half of the proof of Theorem 18, below). Thus one might say that classes tied to $V$ are 'well-behaved'. This contrasts with how little can be proved about classes in general.

THEOREM 18. $\vdash{ }_{A} * T d_{\varphi}(x, y) \rightarrow \operatorname{Rel}(y, \Sigma)$, where $\Sigma$ is any sentence valid in $\boldsymbol{T}_{1}$.

Proof. It is obviously enough to prove Theorem 18 for sentences $\Sigma$ which are axioms of $\boldsymbol{S}+\rho$ or instances of $\boldsymbol{P} \boldsymbol{R}_{1}$. Assume that $T d_{\varphi}(x, y)$.

Let $\sigma$ be an axiom of $S+\rho$. Assume that $\sim \operatorname{Rel}(y, \sigma)$. Then by Theorem $17\left(\exists y^{\prime} \in V\right)\left(W\left(y^{\prime}\right) \wedge \sim \operatorname{Rel}\left(y^{\prime}, \sigma\right)\right)$. Therefore, by Lemma 13(d) 
and Lemma 2(a), we have $\operatorname{Rel}\left(V,\left(\exists y^{\prime}\right)\left(W\left(y^{\prime}\right) \wedge \sim \operatorname{Rel}\left(y^{\prime}, \sigma\right)\right)\right)$. But this leads to a contradiction since $S \subseteq A^{*} / V$ and, as is well-known, one can show within $\boldsymbol{S}$, for each axiom $\sigma$ of $\boldsymbol{S}+\rho$, that $\left(\forall y^{\prime}\right)\left(W\left(y^{\prime}\right) \rightarrow \operatorname{Rel}\left(y^{\prime}, \sigma\right)\right)$.

Let $\Sigma$ be the typical instance (4) of $\boldsymbol{P R}_{1}$. By Lemma 2(a), (b), the statement $\operatorname{Rel}(y, \Sigma)$, which we wish to prove, is equivalent to:

$$
\begin{aligned}
& \left(\forall x_{0}, \cdots, x_{n-1} \in V\right)[\operatorname{Rel}(y, \psi) \rightarrow(\exists u \in V)(\operatorname{Sc}(u) \\
& \left.\left.\wedge x_{0}, \cdots, x_{n-1} \in u \wedge \operatorname{Rel}(u, \psi)\right)\right] .
\end{aligned}
$$

But the latter is an immediate consequence of Theorem 17.

We can now establish in $A^{*} / V$ the result of Theorem 7 that there are arbitrarily large $Q$-sets.

THEOREM 19. (a) $\vdash_{A^{*} / V}(\exists v)(t \in v \wedge Q(v))$.

(b) $\vdash{ }_{A^{*} / V} \sigma \rightarrow(\exists v)(t \in v \wedge Q(v) \wedge \operatorname{Rel}(v, \sigma))$.

Proof. Let $\theta(x)$ be the formula $(\exists u)(x \in u \wedge Q(u))$. What we must show (for (a)) is that $\vdash_{A^{*}}(\forall x \in V) \operatorname{Rel}(V, \theta(x))$. By Theorems 11 and 18,

$$
\vdash_{A^{*}} \operatorname{Td}_{\varphi}(x, y) \rightarrow \operatorname{Rel}(y, Q(V)) ;
$$

and hence, obviously,

$$
\vdash{ }_{A^{*}} T d_{\varphi}(x, y) \rightarrow \operatorname{Rel}(y, \theta(x)) \quad \text { (for every } \varphi \text { ) . }
$$

The desired conclusion is an immediate consequence of (7), by the 'metamathematical principle' following Theorem 16.

If $\theta(x)$ above is replaced by $(\exists u)(x \in u \wedge Q(u) \wedge \operatorname{Rel}(u, \sigma))$, then exactly the same argument establishes (b).

Exactly as we obtained Corollary 8 from 7 one obtains from Theorem 19

Corollary 20. $A^{*} / V$ is essentially reflexive over $\boldsymbol{T}_{1} / V$ and $\boldsymbol{T}_{0}$.

It should be remarked, however, that Theorem 19 is much stronger than Corollary 20. By looking at the proofs of Theorems 11, 18, etc., one easily sees how to obtain a much simplified proof of Corollary 20 .

From the existence of arbitrarily large $Q$-sets one can pass at once within $\boldsymbol{Z F}$ to the existence for every ordinal $\alpha$ of the $\alpha$ th $Q$-set (cf. Theorem 10). However, to make this passage in $A^{*} / V$, as we shall do now, requires some more effort.

$$
\text { Lemma 21: } \vdash_{T_{1}} v \in V \rightarrow[\operatorname{Rel}(V, Q(v)) \longleftrightarrow Q(v)] \text {. }
$$

Proof. Suppose $v \in V$. It is easy to check, using Definition 6 and Lemmas 2(a) and 4(d) that (on the basis of $S$ and so, a fortiori, of $\boldsymbol{T}_{1}$ ) 


$$
w \in z \wedge W(z) \wedge Q(w) \rightarrow \operatorname{Rel}(z, Q(w)) .
$$

From this and Lemma 2(a) and the fact that $W(V)$, one obtains the desired right to left implication.

Since $\boldsymbol{S} \subseteq \boldsymbol{T}_{1} / V$, (8) also holds relativized to $V$. Simplifying by means of Lemma 2(a), (c), since $S c(V)$, this gives

$$
W(z) \wedge \operatorname{Rel}(V, Q(w)) \wedge w \in z \in V \rightarrow \operatorname{Rel}(z, Q(w)) .
$$

Now assume, contrary to the Lemma, that

$$
v \in V \wedge \operatorname{Rel}(V, Q(v)) \wedge \sim Q(v) .
$$

Then, by (9), we see that $W(z) \wedge v \in z \in V \rightarrow \operatorname{Rel}(z, Q(v))$, while by $\boldsymbol{P R}^{*}$ we obtain the contradictory result that

$$
(\exists z \in V)[W(z) \wedge v \in z \wedge \operatorname{Rel}(z, \sim Q(v))] .
$$

LeMma 22. $\vdash_{r_{1}} t, u \in V \rightarrow\left[\operatorname{Rel}\left(V, Q^{\prime}(t, u)\right) \longleftrightarrow Q^{\prime}(t, u)\right]$.

Proof. Lemma 22 can be easily derived from Lemma 21. We shall only give the hint that one should look at Definition 9 and make some simple absoluteness arguments.

THEOREM 23. $\vdash{ }_{A^{*} / V}(\forall t)\left(\operatorname{Ord}(t) \rightarrow(\exists u) Q^{\prime}(t, u)\right)$.

Proof. We must show that

$$
\vdash_{A} * \operatorname{Rel}\left(V,(\forall x)\left(\operatorname{Ord}(x) \rightarrow(\exists u) Q^{\prime}(x, u)\right) .\right.
$$

Since $S \subseteq A^{*} / V$, the principle of transfinite induction holds in $A^{*} / V$. Hence, it suffices to show in $A^{*}$ that, if

$$
\alpha \in V \wedge \operatorname{Rel}\left(V,(\alpha) \operatorname{Ord} \wedge(\forall \beta \in \alpha)(\exists v) Q^{\prime}(\beta, v)\right),
$$

then $\operatorname{Rel}\left(V,(\exists u) Q^{\prime}(\alpha, u)\right)$.

Assume (10). Then, by Lemma 22 and Theorem 18,

$$
T d_{\varphi}(\alpha, y) \rightarrow(\forall \beta \in \alpha)(\exists v \in V) \operatorname{Rel}\left(y, Q^{\prime}(\beta, v)\right) .
$$

and hence, obviously,

$$
T d_{\varphi}(\alpha, y) \rightarrow \operatorname{Rel}\left(y,(\exists w)(\forall \beta \in \alpha)(\exists v \in w) Q^{\prime}(\beta, v)\right) .
$$

Therefore, by the 'metamathematical principle' after Theorem 16,

$$
\operatorname{Rel}\left(V,(\exists w)(\forall \beta \in \alpha)(\exists v \in w) Q^{\prime}(\beta, v) .\right.
$$

From (11) and Theorem 19 it follows easily that $\operatorname{Rel}\left(V,(\exists u) Q^{\prime}(\alpha, u)\right)$. (The needed argument, which we leave to the reader, can be carried out within $A^{*} / V$ on the basis of familiar methods well-known to be valid 
in $S)$.

7. Final remarks. Though we have applied Theorem 16 repeatedly, we appear by no means to have utilized its full strength in the preceding arguments. In all our applications, the formula $\varphi(x, V)$ has been taken to be of the form $\operatorname{Rel}(V, \theta(x))$, or possibly, $\operatorname{Rel}(V, \theta(x)) \wedge W(V)$. As an illustration of an application of Theorem 16 where $\varphi(x, V)$ is a 'higher order proposition' we may mention the following:

In $A^{*}$, we can establish the existence of a class $y$ tied to $V$ such that $\left\langle y, \epsilon_{y}\right\rangle$ and $\left\langle V, \epsilon_{V}\right\rangle$ have the same true sentences. (Indeed for a fixed $z \in V$ we can even demand that elements of $z$ satisfy the same formulas in $\left\langle y, \epsilon_{y}\right\rangle$ as they do in $\left\langle V, \epsilon_{V}\right\rangle$ ). To see this, note that the set $x$ of true sentences of $\left\langle V, \epsilon_{V}\right\rangle$ is a set of natural numbers and hence a member of $V$, and we can easily construct a formula $\varphi(x, V)$ which expresses the fact that $x$ is the set of truths of $\left.<V, \in_{V}\right\rangle$. (The situation when $z$ is given can be handled analogously).

In the argument just sketched, one question needs clarification. In speaking of satisfaction in $\left\langle y, \epsilon_{y}\right\rangle$ we refer to members of the power set $P(y)$ or even of $P P P(y)$. Since these classes are beyond the reach of Theorem 18, how can we be sure our statements about satisfaction have their intended meaning? The answer is to be found in Theorem 17. Since the formula $\psi(y)$ there can also be allowed to refer to classes as large or larger than $y$, we easily see that the classes $P(y), P P(y)$, etc. must exist and be 'well-behaved'. Indeed, by arguing as in the proof of Theorems 16 and 18, replacing mention of $\boldsymbol{R}$ by mention of Theorem 17, one can obtain a kind of iteration of those results, namely the following fact (roughly speaking): If $t \in V$ and $\varphi^{\prime}(t, y)$ and $y$ is tied to $V$, then there exists a class $y^{\prime}$ which is $\varphi^{\prime}$-tied to $y$ by $t$; moreover, for this $y^{\prime}$ we have $\operatorname{Rel}\left(y^{\prime}, \Sigma\right), \Sigma$ being an axiom of $T_{1}$.

Our final remark is that the results of this paper can be extended to various theories with stronger axioms of infinity. If we look at the hierarchy $\boldsymbol{S}, \boldsymbol{Z F}, \boldsymbol{Z M}, \cdots$ of set theories of [4] we see that, roughly speaking, the present paper has dealt with the 'interval' from $\boldsymbol{S}$ to $\boldsymbol{Z F}$. However, it is straightforward to verify that all the numbered definitions and theorems in this paper from Lemma 5 on, together with their proofs, can be imitated in the next 'interval' from $\boldsymbol{Z F}$ to $\boldsymbol{Z M}$, and in higher intervals. To illustrate this point we shall formulate explicitly the counterparts of Corollaries 8, 12 and 20 in the interval from $\boldsymbol{Z F}$ to $\boldsymbol{Z M}$.

$\operatorname{Scm}^{Z F^{\prime}}(x)$ is the formula defined in [4], p. 224 (which means roughly that $x$ is of the form $R(\alpha)$ with $\alpha$ inaccessible). $\quad \boldsymbol{Z F}_{0}$ is the set theory obtained from $\boldsymbol{Z F}$ by adding to it the axiom schema 


$$
\varphi \rightarrow(\exists u)\left(\operatorname{Scm}^{Z \boldsymbol{F}}(u) \wedge x_{0}, \cdots, x_{n-1} \in u \wedge \operatorname{Rel}(u, \varphi)\right)
$$

where $\varphi$ has at most the free variables $x_{0}, \cdots, x_{n-1} \cdot{ }^{(17)} \quad \boldsymbol{Z F}_{1}$ has, in addition to $\varepsilon$, the individual constant $V$. Its axioms are those of $\boldsymbol{Z F}$ together with $\operatorname{Scm}^{Z \boldsymbol{F}}(V)$ and the axiom schema:

$$
x_{0}, \cdots, x_{n-1} \in V \wedge \psi \rightarrow(\exists u \in V)\left(\operatorname{Scm}^{Z F}(u) \wedge x_{0}, \cdots, x_{n-1} \in u \wedge \operatorname{Rel}(u, \psi)\right)
$$

where $\psi$ has no free variables except $x_{0}, \cdots, x_{n-1}$. $A_{0}^{*}$ is the set theory obtained from $A^{*}$ by adding to it the axiom of replacement $\left(\varepsilon_{0}\right)$ of [3], p. 157. As was shown in [3], $A_{0}^{*} / V \subseteq \boldsymbol{Z M}$.

Now, the counterparts of Corollaries 8,12 , and 20 may be stated as follows: $\boldsymbol{Z} \boldsymbol{M}$ is essentially reflexive over $\boldsymbol{Z} \boldsymbol{F}_{1} / V$ and $\boldsymbol{Z} \boldsymbol{F}_{0} . \quad \boldsymbol{Z F}_{1} / V$ is essentially reflexive over $\boldsymbol{Z F}_{0} . \quad A_{0}^{*} / V$ is essentially reflexive over $\boldsymbol{Z F}_{1} / V$ and $\boldsymbol{Z} \boldsymbol{F}_{0}$.

\section{BIBLIOGRAPHY}

1. W. Ackermann, Zur Axiomatik der Mengenlehre, Mathematische Annalen, 131 (1956), 336-345.

2. S. Feferman and R. Montague, The method of arithmetization and some of its applications, North Holland Pub. Co., to appear.

3. A. Lévy, On Ackermann's set theory, Journal of Symbolic Logic, 24 (1959), 154-166.

4. - Axiom schemata of strong infinity in axiomatic set theory, Pacific J. Math., 10 (1960), 223-238.

5. - Principles of reflection in axiomatic set theory, Fundamenta Mathematicae, 49 (1960), 1-10.

6. R. Montague and R. Vaught, Natural models of set theories, ibid, 47 (1959), 219-242.

7. D. Scott, The notion of rank in set theory (abstract), Summaries of talks presented at the Summer institute of Symbolic Logic in 1957 at Cornell University, pp. 267-269.

8. A. Tarski, A. Mostowski and R. Robinson, Undecidable theories, Amsterdam, 1953.

9. H. Wang and R. McNaughton, Les systèmes axiomatiques de la théorie des ensembles, Paris and Louvain, 1953.

UNiversity of CALIFornia, Berkeley

$17 \boldsymbol{Z F _ { 0 }}$ is the theory called $\boldsymbol{Z F} * * *$ in [5]. 


\title{
PACIFIC JOURNAL OF MATHEMATICS
}

\section{EDITORS}

\author{
Ralph S. Phillips \\ Stanford University \\ Stanford, California \\ F. H. BrownelL \\ University of Washington \\ Seattle 5 , Washington
}

A. L. Whiteman

University of Southern California

Los Angeles 7. California

L. J. PAIGE

University of California

Los Angeles 24, California

\section{ASSOCIATE EDITORS}

\author{
E. F. BECKENBACH \\ T. M. CHERRY
} \\ D. DERRY \\ M. OHTSUKA \\ UNIVERSITY OF BRITISH COLUMBIA \\ CALIFORNIA INSTITUTE OF TECHNOLOGY \\ UNIVERSITY OF CALIFORNIA \\ MONTANA STATE UNIVERSITY \\ UNIVERSITY OF NEVADA \\ NEW MEXICO STATE UNIVERSITY \\ OREGON STATE COLLEGE \\ UNIVERSITY OF OREGON \\ OSAKA UNIVERSITY \\ UNIVERSITY OF SOUTHERN CALIFORNIA
}

H. L. ROYDEN

E. SPANIER
E. G. STRAUS

F. WOLF

\section{SUPPORTING INSTITUTIONS}

\author{
STANFORD UNIVERSITY \\ UNIVERSITY OF TOKYO \\ UNIVERSITY OF UTAH \\ WASHINGTON STATE COLLEGE \\ UNIVERSITY OF WASHINGTON \\ AMERICAN MATHEMATICAL SOCIETY \\ CALIFORNIA RESEARCH CORPORATION \\ HUGHES AIRCRAFT COMPANY \\ SPACE TECHNOLOGY LABORATORIES \\ NAVAL ORDNANCE TEST STATION
}

Mathematical papers intended for publication in the Pacific Journal of Mathematics should be typewritten (double spaced), and the author should keep a complete copy. Manuscripts may be sent to any one of the four editors. All other communications to the editors should be addressed to the managing editor, L. J. Paige at the University of California, Los Angeles 24, California.

50 reprints per author of each article are furnished free of charge; additional copies may be obtained at cost in multiples of 50 .

The Pacific Journal of Mathematics is published quarterly, in March, June, September, and December. The price per volume (4 numbers) is $\$ 12.00$; single issues, $\$ 3.50$. Back numbers are available. Special price to individual faculty members of supporting institutions and to individual members of the American Mathematical Society: $\$ 4.00$ per volume; single issues, $\$ 1.25$.

Subscriptions, orders for back numbers, and changes of address should be sent to Pacific Journal of Mathematics, 103 Highland Boulevard, Berkeley 8, California.

Printed at Kokusai Bunken Insatsusha (International Academic Printing Co., Ltd.), No. 6 , 2-chome, Fujimi-cho, Chiyoda-ku, Tokyo, Japan.

PUBLISHED BY PACIFIC JOURNAL OF MATHEMATICS, A NON-PROFIT CORPORATION

The Supporting Institutions listed above contribute to the cost of publication of this Journal, but they are not owners or publishers and have no responsibility for its content or policies. 


\section{Pacific Journal of Mathematics}

\section{Vol. 11, No. $3 \quad$ BadMonth, 1961}

Errett Albert Bishop, A generalization of the Stone-Weierstrass theorem ..........

Hugh D. Brunk, Best fit to a random variable by a random variable measurable with

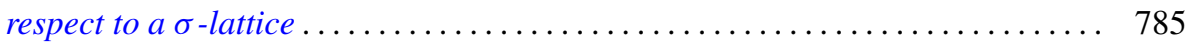

D. S. Carter, Existence of a class of steady plane gravity flows . . . . . . . . . . 803

Frank Sydney Cater, On the theory of spatial invariants ............... 821

S. Chowla, Marguerite Elizabeth Dunton and Donald John Lewis, Linear

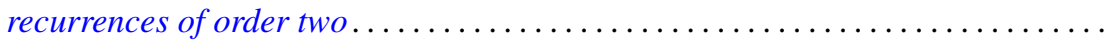

Paul Civin and Bertram Yood, The second conjugate space of a Banach algebra as

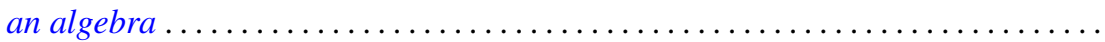

William J. Coles, Wirtinger-type integral inequalities .

Shaul Foguel, Strongly continuous Markov processes ....................

David James Foulis, Conditions for the modularity of an orthomodular lattice ...... Jerzy Górski, The Sochocki-Plemelj formula for the functions of two complex variables.

John Walker Gray, Extensions of sheaves of associative algebras by non-trivial

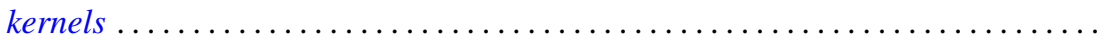

Maurice Hanan, Oscillation criteria for third-order linear differential equations .... 919 Haim Hanani and Marian Reichaw-Reichbach, Some characterizations of a class of unavoidable compact sets in the game of Banach and Mazur .............

John Grover Harvey, III, Complete holomorphs . . . . . . . . . . . . . . . . 961 Joseph Hersch, Physical interpretation and strengthing of M. Protter's method for vibrating nonhomogeneous membranes; its analogue for Schrödinger's

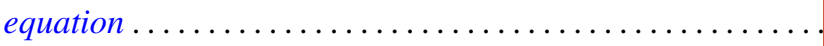

James Grady Horne, Jr., Real commutative semigroups on the plane...

Nai-Chao Hsu, The group of automorphisms of the holomorph of a group...

F. Burton Jones, The cyclic connectivity of plane continua

John Arnold Kalman, Continuity and convexity of projections and barycentric

coordinates in convex polyhedra

Samuel Karlin, Frank Proschan and Richard Eugene Barlow, Moment inequalities of

Pólya frequency functions .

Tilla Weinstein, Imbedding compact Riemann surfaces in 3-space. .

Azriel Lévy and Robert Lawson Vaught, Principles of partial reflection in the set

theories of Zermelo and Ackermann

Donald John Lewis, Two classes of Diophantine equations .....

Daniel C. Lewis, Reversible transformations . . .

Gerald Otis Losey and Hans Schneider, Group membership in rings and

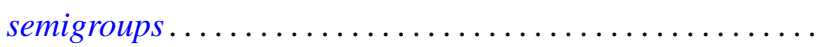

M. N. Mikhail and M. Nassif, On the difference and sum of basic sets of

polynomials.

Alex I. Rosenberg and Daniel Zelinsky, Automorphisms of separable algebras .

Robert Steinberg, Automorphisms of classical Lie algebras .... 